\title{
AUTOEFICACIA Y RIESGO DE RECAÍDA EN DROGODEPENDIENTES
}

\section{SELF-EFFICACY AND RISK OF RELAPSE IN DRUG ADDICTS}

\author{
Jefferson Javier Minda Mina ${ }^{1}$
}

Recibido: 2021-03-12 / Revisado: 2021-04-05 / Aceptado: 2021-05-10 / Publicado: 2021-07-01

Forma sugerida de citar: Minda-Mina, J. J. (2021). Autoeficacia y riesgo de recaída en drogodependientes. Retos de la Ciencia. 5(11), pp. 44-56. https://doi.org/10.53877/rc.5.11.20210701.05

\section{RESUMEN}

Este trabajo expone algunos de los riesgos para la recaída en el consumo de personas drogodependientes. A partir de la teoría cognitivo conductual cuyos postulados plantean que al aprendizaje es la esencia del cambio de conducta. El objetivo de la investigación es analizar la autoeficacia como la capacidad que tienen el ser humano para recurrir a mecanismos de orden cognitivo y no cognitivo para reconocer situaciones adversas y llevar a cabo autónomamente estrategias de superación. El enfoque de esta investigación es cualitativo y de tipo descriptiva. Se encuentra que los principales riesgos de recaída de las personas drogodependientes son: situaciones difíciles de afrontar, diversión y placer, influencia de amigos o pareja que consumen, cercanía de sustancias. Se concluye que las personas drogodependientes deben estar generalmente en compañía de familiares y amigos que influyan positivamente en su conducta, así como, recibir refuerzos terapéuticos secuenciales a fin de fortalecer sus niveles a autoeficacia, hasta un estado de recuperación integral. Cabe señalar que, cuando una persona ha sido drogodependiente por lo general no alcanza niveles de recuperación total, por tanto, la observación será permanente.

Palabras clave: autoeficacia, consumo, recaída, riesgo de consumo, drogodependencia.

\section{ABSTRACT}

This work exposes some of the risks for the relapse in the consumption of drug addicts. From the cognitive behavioral theory whose postulates state that learning is

\footnotetext{
1 Psicólogo Clínico. Maestrante en Intervención Psicológica en Adicciones en la Facultad de Ciencias Psicológicas de la Universidad Central del Ecuador. Ecuador. Email: jjminda@uce.edu.ec / ORCID: https://ordcid.org/0000-00016618-8243
} 
the essence of behavior change. The objective of the research is to analyze selfefficacy as the ability of the human being to resort to cognitive and non-cognitive mechanisms to recognize adverse situations and autonomously carry out strategies for improvement. The focus of this research is qualitative and descriptive. It is found that the main risks of relapse of drug addicts are difficult situations to face, fun and pleasure, influence of friends or partner who consume, proximity of substances. It is concluded that drug addicts should generally be in the company of family and friends who positively influence their behavior, as well as receive sequential therapeutic reinforcements in order to strengthen their levels of self-efficacy, up to a state of comprehensive recovery. It should be noted that when a person has been drug dependent, they generally do not reach full recovery levels, therefore, the observation will be permanent.

Keywords: self-efficacy, use, relapse, risk of use, drug dependence.

\section{INTRODUCCIÓN}

La autoeficacia ha sido considerada como uno de los componentes explicativos de la conducta, puesto que, se la relaciona con el hecho de adquirir, mantener y organizar el cambio de los comportamientos. Así como lo menciona Bandura (1997), describiendo la forma en la que "el sujeto se relaciona en el proceso cognitivo con aspectos del condicionamiento clásico, el condicionamiento operante y el aprendizaje vicario, es así como los procesos de cognición determinan la modificación de conducta (p. 34)

Así también como lo dice Roca (2002) en su artículo sobre la "Autoeficacia y su valor para la psicoterapia cognitivo conductual", en el cual describe como:

La autoeficacia tiene sus inicios en los estadios tempranos del ciclo vital, de esa manera el individuo trata de conservar la autoimagen, la persona en todo el ciclo vital es persuadida, es "sometida a una serie de influencias que hacen de la autoeficacia una dinámica formación que no permanece estática en modo alguno en tanto la persona interactúa constantemente con los retos y avatares de la vida cotidiana. (p. 198)

Aun así, hay muchas opciones que las personas pueden realizar con un grado de éxito, solamente que no las realizan por el miedo a fracasar o por no tener los incentivos para cumplir los propósitos individuales.

Lo expuesto sugiere que los niveles de autoeficacia son orientaciones para identificar posibles conductas. De ser el caso, "la evaluación oportuna de la autoeficacia es una alternativa apropiada para evitar conductas no deseadas" (López, 2002, p. 34).

Entendiendo que la autoeficacia pone énfasis en la autopercepción del sujeto previo a la acción ante determinada situación, podría decirse, que una persona con elevados niveles de autoeficacia reconocería autónomamente la presencia de deseos por consumir sustancias no permitidas o realizar acciones antisociales que podrían afectar a sí mismo y a los demás en cuyo caso recurriendo a cualidades como capacidad de decisión, automotivación y autodeterminación.

Según indica Bandura (1982), conscientemente desarrollaría acciones qué le alejen de tales intereses:

Cabe señalar que el sujeto desarrolla su capacidad perceptiva frente a influencias internas y externas y a partir de sus posibilidades de autocontrol, toma las decisiones más idóneas desde su perspectiva. Cuando los niveles de auto 
eficiencia son elevados y le permiten al sujeto distinguir positivas y negativas, éste será capaz de alejarse de lo negativo. Lo cual, a más de evitar una recaída en situaciones de consumo, fortalece su autoestima y potencia su autocontrol.

Cabe mencionar que la recaída muchas veces constituye un fracaso en el proceso de recuperación. Cuando un paciente recae en el consumo de drogas se presentan varios comportamientos, entre otros, se destacan que se ralentiza el proceso de recuperación, se presenta rechazo del paciente hacia el terapeuta, existen comportamientos de inhibición e incluso abandono del tratamiento. (Ledwidge, 1978)

Dentro del modelo de prevención de recaída desde la teoría cognitivo conductual según Ledwidge (1978) se la considera como una bifurcación cuando "uno de los caminos termina aportando al sujeto una experiencia negativa que ayuda para que se aleje del contexto de consumo", por otro lado, la recaída podría constituir una experiencia en la que el sujeto se sienta incapaz de volver a abandonar el consumo.

Considerando que la recaída no se encuentra exenta en ninguno de los casos en los que el sujeto culmine un proceso de recuperación, hay que tomar en cuenta que:

Existen factores que pueden alertar al sujeto y su contexto de una posible recaída, dentro de estos se encuentran los niveles bajos de autoeficacia, que básicamente son las creencias de poca capacidad para afrontar situaciones adversas, además del contacto o vuelta al contexto de consumo. (Olivari Medina y Urra, 2007, p. 12)

Demostrando así, como los bajos niveles de autoeficacia aumentan la probabilidad de tener una recaída. Este trabajo se desarrolla desde un enfoque descriptivo, basado en detallar los hallazgos a cerca de la autoeficiencia y su relación con las recaídas en drogodependientes, para ello se ha realizado un amplio estudio teórico, que sirvió de base para el análisis y dilucidación de esta problemática, cumpliendo con el objetivo de analizar e interpretar cómo influye la autoeficacia en la recaída del consumo de drogas dentro de los hallazgos identificados.

Aspectos a considerar para la revisión teórica: bibliografía perteneciente al área de salud en relación al consumo de drogas; datos bibliográficos de hace más de cinco años que sean relevante en la investigación o considerada clásica, esto considerando que: "Las técnicas e instrumentos recogen datos sobre los temas y subtemas de las categorías de análisis" (Castillo-Bustos, 2021, p. 59); Datos estadísticos sobre adicciones relevantes dentro de la investigación; Para los criterios de exclusión se ubican los siguientes: bibliografía de más de cinco años que no sea tomada como relevante para la investigación, casos clínicos de adicciones que no tengan seguimiento terapéutico con base científica, revistas con artículos que cuenten con un carácter científico.

\section{DESARROLLO}

\section{La autoeficacia}

Al hablar de autoeficacia las personas tienden a separar sus vocablos e identificar por separado su significado dando como resultado interpretaciones poco adecuadas den relación con su concepto real, de similar manera sucede en el estudio de 
distintas ciencias, específicamente dentro de la literatura sobre psicología, creando así una amplia discusión de su significado.

Es por ello por lo que nos remontamos al concepto planteado por Olivari y Urra (2007) quienes entienden a la autoeficacia como: La creencia personal del individuo para dar respuesta situaciones que considera estresante. Este hará uso de sus habilidades personales y en la medida en que afronte positivamente la situación, su nivel de eficiencia aumentará, pudiendo aprovechar este aprendizaje en el ámbito académico, social, laboral entre otros (p. 10)

También encontramos el concepto de Melo y Castanheira (2010) quienes indican como "la autoeficacia surge en un intento de explicar los cambios de conducta dentro de un modelo unificado", coincidiendo con lo dicho por Bandura (1982) en su libro Mecanismo de autoeficacia en la agencia humana, en donde muestra como esta conducta se basa en la motivación refiriéndose a la autoeficacia como "reacciones espontáneas debido a múltiples estímulos, una conducta motivada incluye un objetivo, meta o plan"(p.38)

Logrando así ver como se genera un mecanismo cognitivo en donde las creencias se encuentran ligadas a la autoeficacia incidiendo dentro de los procesos cognitivos, emocionales, motivaciones y conductuales. Por tanto, el individuo desarrollará convicción y seguridad en su propia capacidad de superar dificultades. Lo cual se refuerza en las relaciones sociales. Téngase en cuenta que, "El intercambio de ideas, experiencias, conocimientos, emociones, sentimientos y pasiones" (Castillo-Bustos, 2015, p. 199), conlleva al desarrollo integral del ser humano.

\section{Componentes de la autoeficacia}

Para Bandura (1982) la expectativa de autoeficacia se relaciona con las creencias que tiene el sujeto sobre la habilidad o capacidad que posee para realizar acciones. Así como la expectativa de resultado se relaciona con que dicha acción tomada se oriente a un resultado específico. Los dos componentes: la expectativa de autoeficacia y la expectativa de realización inciden en que las habilidades del sujeto al momento de afrontar situaciones que se consideren de alto riesgo, estas creencias se ven influenciadas por las mismas que el individuo tenga a nivel social, cultural e individual. Entendiendo a la autoeficacia como un mecanismo cognitivo se puede evidenciar que a través de la teoría del aprendizaje social se puede cambiar conductas o ejercer influencia en la motivación.

Según Abaitua y Ruiz (1990) en su artículo a cerca las expectativas de la autoeficacia resaltan cómo se puede verificar que los componentes de esta conducta pueden ser manipulados por la persuasión en los individuos, "estos componentes se pueden considerar desde la necesidad del individuo de cumplir expectativas, de verificar que las capacidades personales surgen cuando se debe enfrentar una problemática", es así que dichas expectativas son parte de los componentes de la autoeficacia, creando expectativas de autoeficacia y expectativa de resultado.

Hay que resaltar también cuán importante es comprender el funcionamiento cognitivo de la autoeficacia ya que es la dinámica con la que se lleva a cabo ideas, pensamientos y acciones; permiten que se fortalezca la toma de decisiones del individuo ya sea de manera positiva o negativa, en este sentido. Ya que según dicen algunos autores "La adecuada percepción de la autoeficacia va a generar un correcto autocuidado, también lo que se espera de la autoeficacia tiene una relación 
negativa con reacciones ansiosas o depresivas y tienen una asociación positiva con persistir en la tarea" (Pereyra, Ronchieri, Rivas, y Trueba, 2018, p. 311).

Demostrando así que para comprender el funcionamiento de la autoeficacia es necesario enfrentar al individuo a situaciones estresantes, ya que esta la única manera en la cual se puede verificar el comportamiento para resolver problemáticas, las creencias sobre las propias capacidades darán la sensación de autonomía y buena toma de decisiones, esto permite generar expectativas de resultado.

Según Pereyra, Ronchieri, Rivas, y Trueba (2018) "para que las creencias de lo que se ha planificado salga con éxito es necesario que los resultados que se obtienen sean óptimos" ( $p$. 311) percibiendo, así como se enfoca en gran medida la necesidad de fortalecer la autoeficacia interpersonal para que aparte de tener convicción sobre sí mismo también se aplique influencia positiva sobre las personas del medio que rodea al individuo.

\section{Características de la autoeficacia}

Según Roca (2002), en su artículo a cerca de la "Autoeficacia: su valor para la psicoterapia cognitivo conductual "detalla cómo esta conducta es un concepto expuesto por Bandura, "en el cual se expresa que es una reconceptualización de aprendizajes y motivaciones las cuales son de gran influencia para la activación conductual" (p. 196).

Según Roca (2002), de esta manera se evidencian comportamientos en los cuales los individuos a través de las expectativas de eficacia personal demuestran:

- Si las conductas de afrontamiento serán iniciadas o inhibidas.

- La cantidad de esfuerzo que será puesto en alguna actividad o propósito.

- La cantidad del tiempo en que se persistirá para lograr el objetivo de cada individuo y la búsqueda de desafíos y experiencias que surgen en la búsqueda de alcanzar propósitos personales. (p. 197)

De esa manera se identifica que un individuo con alta sensación de eficacia será capaz de:

- Iniciar proyectos en los cuales se sienta competente.

- Implicarse en nuevas actividades las cuales sienta capacidad para lograrlas.

- Comprometerse con nuevos propósitos a pesar de los cambios que pueda generar.

- Tener una filosofía de vida progresista y optimista (p. 197).

Por otro lado, en este artículo también podemos observar cómo Roca (2002), indica que el individuo con bajas expectativas de eficacia personal va a presentar las siguientes características:

- Tendencia a ser conservador o reservado.

- Evitar los cambios.

- Iniciar pocas actividades por iniciativa propia.

- Las personas presionan e influyen en la toma de decisiones del individuo.

- Más atención a los riesgos que a las oportunidades (p. 197).

\section{Factores de la autoeficacia}

Considerando que todos los procesos psicológicos individuales o sociales pueden influir en el nivel de autoeficacia y que ésta a su vez, puede determinar el cambio en la conducta, Bandura (1977) menciona cuatro factores: 1) Logro de ejecución, 2) experiencia vicaria, 3) persuasión verbal, 4) activación psicológica y emocional.

El logro de ejecución se basa en experiencias reales: cuanta mayor cantidad de éxito perciba el individuo, aumentan las expectativas positivas y se reducen las expectativas de fracaso. Se incluyen también los fracasos repetidos que han sido 
anulados por esfuerzos posteriores, mismos que le otorgan al sujeto una percepción automotivada. De esta forma, el individuo entiende que las circunstancias difíciles, pueden ser superadas con esfuerzo continuo. Este factor contribuye a la formación, consolidación y por último, el mantenimiento de la autoeficacia. (Roca, 2002, p. 1989)

El segundo factor es la experiencia vicaria, se manifiesta cuando el sujeto, ya sea por observación o imaginación, percibe que sus iguales afrontan situaciones que para él son difíciles, procede a persuadirse y se sitúa en una posición de: "si él puede, yo puedo". Termina modificando la autoeficacia percibida, tomando en cuenta que es el resultado de una interacción con el ambiente.

Es importante mencionar que, "las experiencias vicarias no tienen tanta influencia como las experiencias directas, aun así, son capaces de producir cambios a largo plazo y se determina su influencia debido a las consecuencias derivadas de la elección de comportamiento del individuo" (Roca, 2002, p. 196).

El tercero está dentro de la persuasión verbal, "es la manera más amplia de influir en otra persona, así se manifiesta a través de la aplicación de sugestión verbal enfocada en hacer creer al sujeto que puede controlar o manejar lo que teme" (Roca, 2002, p. 199).

Las expectativas de autoeficacia que se generan aquí son muy débiles, no se basan en ninguna experiencia real en la que el individuo puede recurrir para solventar toda la información que le están brindando.

El cuarto factor tiene enfoque en los estados fisiológicos, también llamado de activación psicológica y emocional.

Se refiere a informar al sujeto de su competencia, procurando evitar o disminuir las frecuentes y duraderas activaciones emocionales, puesto que durante dichos lapsos de tiempo al sujeto se le dificulta la acción y puede creer que es poco competente, disminuyendo las expectativas de autoeficacia, siendo así, el sujeto se percibirá como más eficaz cuando sus niveles de activación emocional se encuentren en niveles normales, "situaciones que son percibidas como un reto a la capacidad personal y movilizan a la acción, son estados emocionales que tienden a confirmar e incentivar las sensaciones de autoeficacia personal". (p. 200)

Es de relevancia mencionar que el cambio conductual no es el que genera la percepción de autoeficacia, sino que esta se construye a través del juicio que el sujeto adquiere por medio de sus interpretaciones cognitivas en base a su propia experiencia o en dirección externa, en la que se adopta una creencia de su propia habilidad.

Ventajas de la autoeficacia

Es indispensable que se establezca un apoyo constante en la vida del individuo y que se otorgue un pensamiento de que puede realizar varios retos y resolverlos, ya que la realidad es que las personas tienen más valor en las actividades que brindan un reto y permiten que sus habilidades crezcan, así como lo demuestra Rivero (2005):

La generación de la autoeficacia se establece en otorgar al individuo la habilidad de crear estrategias o nuevas ideas para resolver problemáticas y que una vez que las lleve a cabo funcionen para el bien de él y de su familia, esto mejorará su autoestima y asertividad para resolver conflictos y mejorar la comunicación. (p. 21)

Cabe recalcar que dentro de la teoría de la autoeficacia existen ventajas, la primera es que constituye una revisión amplia e integradora sobre sí mismo y que además permite ser comprobada a través de la medición de independencia de la 
ejecución, esto a través de la revisión propia de cada propuesta de expectativas específicas y así se puede identificar hasta que medida los acontecimientos que han brindado aprendizaje se han procesado desde la cognición y cuando nos servirán como un punto de referencia para predecir futuras conductas.

Para López, Salvador, y Verdejo (2002) la segunda ventaja es que las expectativas de autoeficacia pueden influir en la elección de conductas, dando como resultado que de esa manera se pueda apreciar que existen pensamientos en el individuo sobre las consecuencias de cada acción y, por lo tanto, exista la decisión de elegir o no cualquier comportamiento (p. 36-7).

Para Melo y Jaimes (2015) es indispensable estar pendiente de las emociones y conductas que no resulten impulsivos.

En un estudio realizado con niños se logró identificar que los varones se perciben con mayor autoeficacia, y ante esto se halla que la condición es que se debe a cumplir las expectativas hacia los demás, en ese sentido es una ventaja poder reconocer la necesidad de estar en contacto con diferentes grupos y que el modelamiento también sea de gran ayuda para la resolución de conflictos. La exigencia que se planteen los individuos también va a repercutir en la autoeficacia, se identifica que en cuanto a los resultados de los infantes de los colegios públicos tienen más altos niveles de autoeficacia que los de los alumnos de los colegios privados (p. 123).

\section{Autoeficacia y riesgo de consumo}

La autoeficacia percibida se relaciona con las creencias sobre la propia capacidad que posee el sujeto y de la que está consciente para organizar y ejecutar acciones que le servirán a futuro para el manejo de situaciones estresantes.

La autoeficacia antes mencionada, se relaciona con la autoeficacia de resistencia, misma que permite al sujeto que se encuentra en abstinencia del consumo de drogas, rechazar la posibilidad de acceder a la sustancia ante la presencia de situaciones de riesgo (ej. reuniones de amigos, juntas de trabajo, momentos de angustia y preocupación). Cuando el individuo supera con éxito las situaciones antes mencionadas, los niveles de autoeficacia aumentan, generando un control de las conductas negativas. (Hinojosa, Alonso, y Castillo, 2012, p. 20)

La decisión última de consumir o no, está en función de las expectativas de autoeficacia y del resultado que tiene la persona en el contexto situacional en que se encuentra. Hay que tomar en cuenta como "los efectos anticipados de una conducta son los que parecen importantes a la hora de determinar si esta conducta se realiza o no" (Melo y Castanheira, 2010, p. 658).

De esta forma podemos observar como es que las creencias que se tiene sobre uno mismo influyen en la autoeficacia, la influencia está enfocada en el modo de pensar, sentir, la motivación y la conducta, "de esa manera si un individuo ha generado la convicción en su propia capacidad de superar retos entonces pensará que los puede superar, tendrá seguridad y habrá que continuar adelante en la planificación de las acciones necesarias para conseguir su propósito" (Melo y Castanheira, 2010).

En relación al riesgo de consumo se identifica como al que lo menciona Grau, y otros (2012), donde afirmaban lo siguiente:

Los altos niveles de autoeficacia como un factor protector individual que puede ayudar al manejo eficaz de los acontecimientos estresantes para el sujeto, siendo así esta ejerce un papel importante dentro de la prevención de riesgo de recaída, 
puesto que si existe más alto nivel de autoeficacia la probabilidad de consumo es bajo. (págs. 115-122)

\section{Recaída y modelo de prevención de recaída}

Tomando en cuenta que el tratamiento para las personas que abusan de sustancias psicoactivas no termina y que es un seguimiento constante, cuando las personas con esta enfermedad asumen el encontrase bien, el tener control sobre su consumo y asumirlo como dado por terminado su tratamiento, uno de los sucesos que ocurren son las recaídas que no solo aparece cuando se termina el proceso de rehabilitación, sino que se da en cualquier momento del tratamiento, mucho más durante los primeros meses donde hay un aumento de recaídas "10 veces más en consumidores de alcohol y 14 veces más en consumidores de heroína"(UNODC, 2017).

Para Hendershot, Witkiewitz, George y Marlatt (2011) se identifica a las recaídas como:

El efecto que va a sufrir el sujeto al mostrarse frente a una acción que genere un riesgo de consumo y sea incapaz de responder con estrategias adecuadas y finalmente rompe su abstinencia, consecuentemente no la mantiene puesto que regresa a sus viejos patrones de consumo. Según algunos autores considera que la recaída podría ser tomada como el punto que dista entre la culminación de un proceso de rehabilitación y el regreso a un contexto de consumo, teniendo siempre en cuenta que pude ocurrir también en cualquier momento del proceso del tratamiento, pero lo que tratan aquí es explicar es que cuando la recaída se suscita, se la considera como un indicador que impide el proceso de mantenimiento de la abstinencia, manifestándose además en afectaciones del sujeto a nivel físico, individual, social, familiar, laboral y económico.

El modelo cognitivo-conductual de prevención recaída en el que Marlatt y Gordon (1985) mencionan que se intenta comprender la recaída desde un marco conceptual en el que esta constituye, "es el resultado de procesos cognitivos, de una exposición a contextos ambientales de riesgo, los mismos que se muestran indicadores antes de que se suscite la recaída". Se diferencia de los modelos tradicionales porque en estas posturas se relaciona una recaída con el fracaso de sostener el deseo de no consumir como la única razón por la que se puede presentar este cambio en el sujeto.

Para Marlatt y Gordon (1985) el modelo de prevención de recaída fue quien permitió generar una alternativa integral, flexible y además optimista frente a la experiencia de la recaída, puesto que considera que constituye la bifurcación entre las que el sujeto puede tomar la alternativa de reincidir en un contexto de consumo y mantenerse. También existe la alternativa de que este regreso al contexto de consumo pueda darse durante un lapso de tiempo y después el sujeto vuelva a corregir el abuso problemático de consumo y esto genere una nueva motivación para obtener un comportamiento deseado en el que el sujeto aproveche las oportunidades de cambio nuevamente.

\section{Características de las recaídas}

Existen indicadores que preceden la misma, en este caso Witkiewitz \& Marlatt, (2004) mencionan que los lapsos, son el momento en el que el sujeto vuelve al comportamiento que estaba ligado al consumo, de hecho, en el mayor de los casos este antecesor trae inevitablemente la recaída (p. 111).

Explicando al consumo puntual como la disponibilidad de la sustancia, mientras que la recaída es el consumo reiterado asociado a reforzadores positivos y 
negativos, búsqueda de consecuencias agradables o eliminación de consecuencias desagradables, es la vuelta al consumo continuado y compulsivo de la droga, la vuelta a su patrón de consumo anterior.

Por lo tanto, este lapso puede servir como el puente en el que el sujeto elige no volver al contexto de consumo, en este caso el sujeto experimenta un aprendizaje positivo al no retornar al contexto de consumo:

Un factor importante de mencionar es la situación de alto riesgo que se considera como cualquier contexto que le proporcione vulnerabilidad al sujeto para mantener su comportamiento objetivo de abstinencia, estos pueden direccionarse hacia el ámbito emocional como en el caso de un sentimiento negativo ligado a un nivel bajo de autoeficacia. (Villamarín, 1990, p. 55)

Se puede trasladar al ámbito ambiental, pueden ser situaciones ligadas a experiencias anteriores; además en el ámbito fisiológico, cuando existe una abstinencia aguda. Cabe mencionar que "las situaciones de alto riesgo varían, considerando la individualidad del sujeto y su experiencia en el contexto de consumo" (Baker, Piper, McCarthy, Majeskie, y Fiore, 2004)

Además, las situaciones de alto riesgo que son más frecuentes son las que se relacionan con el ámbito emocional (Brandon, Irvin, \& Litvin, 2007) inherentemente estas se individualizan y no están marcadas para suceder en algún tiempo exacto, ni bajo un ambiente determinado o en un estado emocional particular, sin embargo, no se puede desplazar la importancia de la percepción del sujeto en base a su experiencia a lo largo de su historia de abuso de consumo.

Una reacción a la situación de alto riesgo son los lapsos, los mismos que pueden tener dos alternativas de acción, esto dependerá de la capacidad que tenga el sujeto de responder con afrontamientos que favorezcan el mantenimiento de abstinencia, la autoeficacia se transforma en una importante construcción cognitiva que va a ser utilizada en "la guía de acciones para la promoción de la salud mental y física así como también la prevención en el abuso de estupefacientes" (Melo y Castanheira, 2010, p. 659)

Si bien las recaídas conllevan a que una persona regrese a conductas anteriores, esta situación está mediada por la calidad de los procesos de recuperación, así como también por el seguimiento control que se realice después del tratamiento.

\section{Recaída y autoeficacia}

El manejo adecuado de situaciones de alto riesgo fortalece el desarrollo positivo y mantenimiento de autoeficacia percibida en el sujeto, puesto que el reaccionar con un afrontamiento adecuado evitará que el sujeto reinicie un consumo.

Otra alternativa de reacción negativa ante una situación de alto riesgo y provocando una recaída, afecta de igual forma al nivel de la autoeficacia del individuo puesto que guardará una experiencia negativa de fracaso ante un momento de crisis para el retorno al contexto de consumo. (Larimer, Palmer, y Marlatt, 1999)

En tal sentido, incidir en el desarrollo de la autoeficacia durante los tratamientos, posibilitará que la persona sea capaz de conocerse, valorar sus cualidades y capacidades y a partir de su educabilidad sea capaz de aprender intencionalmente para favorecer la concreción de comportamientos favorables en torno a sí mismo y su contexto.

Sin embargo es importante hacer hincapié de que en caso de existir una recaída después de un fracaso ante el manejo de una situación de alto riesgo, las creencias 
y actitudes que presente el sujeto ante el acontecimiento también podrían terminar favoreciendo al trabajo de fortalecer o establecer una autoeficacia en el sujeto, puesto que la experiencia negativa de fracaso podría terminar constituyendo un lapso en el que el sujeto tome el camino de reiniciar el comportamiento objetivo inicial de abstinencia.

La finalidad del desarrollo de la autoeficacia es generar en el individuo la capacidad de elegir la mejor opción para resolver una dificultad y fortalecer la convicción de sus propias capacidades, para Villamarin (1990) es necesario reconocer que el aprendizaje vicario permite desarrollar estrategias en los individuos para afrontar problemas, pero no los vuelve parte de sí mismos y no perdura, en cambio la autoeficacia genera ejecución de conductas y no solo el modelado simple.

La importancia de generar autoeficacia en cada individuo demuestra que la resolución de problemas tiene su base en la autoestima y en la calidad de las relaciones sociales del sujeto.

Tal como lo señala Roa (2019) la familia es un pilar necesario en la sociedad y vínculo de las personas, la familia es el primer apoyo, y se comprende que la adicción no solamente existe en hijos sino también en padres, es por tanto importante considerar que el componente social es indispensable para establecer alianzas y mejorar el vínculo en la familia. las personas drogodependientes deben estar generalmente en compañía de familiares y amigos que influyan positivamente en su conducta, así como, recibir refuerzos terapéuticos secuenciales a fin de fortalecer sus niveles a autoeficacia, hasta un estado de recuperación integral (p. 7).

El factor cognitivo es importante para comprender el desarrollo de las adicciones y la relación de la autoeficacia con el riesgo de recaídas, para López, Salvador, Verdejo, y Cobo (2002):

Las cogniciones y conductas frente al consumo tienen que ver con las creencias de los efectos que produce el uso de drogas, así que la sensación de eficacia va a iniciar como una conducta de consumo en búsqueda de mejoría al creer que es un comportamiento alternativo, después de evidenciar que es un error buscar conductas que empeoren el estado del sujeto es donde urge la conciencia de enfermedad, por tanto, si se da la rehabilitación esta va a depender del fortalecimiento de las habilidades y alternativas de afrontamiento que genere el paciente. (p. 37)

En cuanto a la toma de decisiones sobre consumir tiene relación con la generación de expectativas sobre la autoeficacia, así como también el resultado que obtiene el paciente dependiendo del contexto en que se halle, "el punto clave en la autoeficacia es tener la capacidad de control sobre la drogodependencia, cuanto más largo sea el periodo entonces va a ser mejor la percepción de uno mismo sobre el autocontrol y por tanto se da el fortalecimiento de la autoeficacia" (López, Salvador, y Verdejo, 2002, p. 37), ante esta afirmación se destaca también que los factores de protección deben ser óptimos ya que la familia sirve de apoyo en todo el proceso de rehabilitación y evitar el riesgo de recaídas.

\section{CONCLUSIONES}

Se resalta la importancia que tiene el evaluar los niveles de autoeficacia durante un proceso de recuperación, esto debido a que un nivel alto de autoeficacia nos servirá como factor protector ante una situación de alto riesgo con respecto a la recaída, cabe mencionar que un trabajo post tratamiento enfocado en optimizar los niveles 
de autoeficacia también terminaría favoreciendo al sujeto, puesto que ya se encuentra dentro de un contexto apartado de consumo, entonces la autoeficacia podría mantener niveles altos.

Todos los estudios e investigaciones presentados en el texto han demostrado que las puntuaciones altas en el nivel de autoeficacia puede ser el punto referente para identificar la seguridad o habilidad de afrontamiento que posee el sujeto para así tener una visión de cómo se podría fortalecer la autoeficacia y que en el sujeto disminuya el riesgo de recaída, si esta evaluación se realiza mientras se encuentra en un proceso de recuperación, la puntuación serviría para contrastar con estado del sujeto en relación a la fase de cambio en la que se encuentre y así poder intervenir en favor de fortalecer el nivel de autoeficacia.

Las experiencias positivas que posea el individuo ante una situación de alto riesgo, al no acceder al consumo el sujeto fortalecerá su nivel de autoeficacia, pero hay que tener en cuenta la integralidad del sujeto, puesto que para que sus niveles de autoeficacia aparezcan como un factor que beneficie la prevención de recaída, el sujeto formara su juicio a través de la evaluación previa de momentos, lugares y personas que puedan generar riesgo para el sujeto y siendo así, este reconocimiento previo proporciona al sujeto una visión más amplia de su propia capacidad y límites, finalmente aportando de forma positiva al desarrollo de autoeficacia.

Por tanto, se determina que como conclusión las situaciones estresantes de aquellos sujetos en riesgo, dependiendo del ambiente donde se encuentren, pueden servirles para superar la situación y ser una experiencia positiva en su vida, así como también ser un lapso para volver a tomar acciones que mejoren su vida.

\section{REFERENCIAS BIBLIOGRÁFICAS}

Abaitua, B., \& Ruiz, M. (1990). Rev. De Psicol. Gral. Y Aplic, Vol. 43. Expectativas de autoeficacia y de resultado en la resolución de tareas de anagramas, 4552.

Baker, T., Piper, M., McCarthy, D., Majeskie, M., \& Fiore, M. (2004). La motivación de la adicción reformulada: un modelo de procesamiento afectivo de refuerzo negativo. Psychol Rev. Vol. 111, núm. 1, 33-51.

Bandura, A. (1977). Autoeficacia: Hacia una teoría unificadora del cambio de comportamiento. Obtenido de Psychological Review. Vol. 84, núm. 2, pp. 191 215: https://doi.org/10.1037/0033-295X.84.2.191

Bandura, A. (1982). Mecanismo de autoeficacia en la agencia humana. Obtenido de Psicólogo estadounidense, Vol. 37, núm. 2, pp. 122-147: https://doi.org/10.1037/0003-066X.37.2.122

Brandon, T., Irvin, J., \& Litvin, E. (2007). Prevención de recaídas y recaídas. Annu Rev Clin Psychol. Vol. 3.

Castillo-Bustos, M. R. (2021). Técnicas e instrumentos para recoger datos del hecho social educativo. Retos de la Ciencia. 5(10), pp. 50-61.

Castillo-Bustos, M. y Montoya-Rivera, J. (2015). Dinámica ideo-espiritual de la formación estético-pedagógica del docente. Alteridad, 10(2), pp. 190-204.

Grau, L., Roncero, C., Daigre, C., Gonzalvo, B., Bachiller, D., Rodríguez, L., . . . Casas, M. (2012). Factores de riesgo de recaída en pacientes drogodependientes tras desintoxicación hospitalaria. Adicciones, Vol. 24, 115122. 
Hendershot, C., Witkiewitz, K., George, H., \& Marlatt, G. (2011). Prevención de recaídas para conductas adictivas. Política anterior de tratamiento de abuso de sustancias. Revista BMC. Vol. 6, núm. 17.

Hinojosa, L., Alonso, M., \& Castillo, Y. (2012). Autoeficacia percibida y consumo de alcohol en trabajadores de la salud. Rev Enferm Inst Mex Seguro Soc. Vol. 20, núm. 1, 19-25.

Larimer, M., Palmer, R., \& Marlatt, G. (1999). Prevención de recaídas: una descripción general del modelo cognitivo-conductual de Marlatt. Obtenido de Alcohol Res Health. Vol. 23, núm. 2, pp. 151-160: https://www.ncbi.nlm.nih.gov/pmc/articles/PMC6760427/

Ledwidge, B. (1978). Modificación cognitiva del comportamiento: ¿un paso en la dirección equivocada? Obtenido de Psychological Bulletin, Vol. 85, núm. 2, pp. 353-375: https://doi.org/10.1037/0033-2909.85.2.353

López Torrecillas, F. (2002). Autoeficacia y consumo de drogas. Obtenido de Dialnet: https://dialnet.unirioja.es/servlet/articulo?codigo $=2518067$

López-Torrecillas, F., Salvador, M., \& Verdejo, A. (2002). Autoeficacia y Consumo de Drogas: Una Revisión. Psicopatología Clínica, Legal y Forense, Vol. 2., 33-51.

López-Torrecillas, F., Salvador, M., Verdejo, A., \& Cobo, P. (2002). Autoeficacia y Consumo de Drogas: Una Revisión. Psicopatología Clínica, Legal y Forense, Vol. 2, 33-51.

Marlatt, G., \& Gordon, J. (1985). Prevención de recaídas: estrategias de mantenimiento en el tratamiento de conductas adictivas. Nueva York: Guilford Press.

Melo, D., \& Castanheira, L. (2010). Autoeficacia y actitud hacia el consumo de drogas en la infancia: explorando los conceptos. Obtenido de Revista Latinoamericana de Enfermagem, Vol. 18: https://doi.org/10.1590/S010411692010000700024

Melo, D., \& Castanheira, L. (2010). Autoeficacia y actitud hacia el consumo de drogas en la infancia: explorando los conceptos. Obtenido de Revista Latinoamericana de Enfermagem, Vol. 18: https://doi.org/10.1590/S010411692010000700024

Melo, D., \& Jaimes, M. (2015). Autoeficacia, actitud hacia el consumo de drogas y salud percibida de niños escolarizados. Obtenido de Scielo.org: http://www.scielo.org.co/pdf/hpsal/v20n2/v20n2a09.pdf

Olivari Medina, C., \& Urra, E. (2007). Autoeficacia y Conductas de Salud. Obtenido de Scielo. Ciencia y enfermería: https://scielo.conicyt.cl/scielo.php?script=sci_arttext\&pid=S071795532007000100002

Pereyra, C., Ronchieri, C., Rivas, A., \& Trueba, D. (2018). Autoeficacia: una revisión aplicada a diversas áreas de la psicología. Ajayu Órgano de Difusión Científica del Departamento de Psicología, vol. 16, núm. 2, 299-325.

Rivero, J. (2005). Políticas educativas y exclusión: sus límites y complejidad. Obtenido de Revista Iberoamericana sobre Calidad, Eficacia y Cambio en Educación, vol. 3, núm. 2: https://www.redalyc.org/pdf/551/55103204.pdf

Roa, M. (2019). Cómo afectan las conductas adictivas en el entorno familiar y cómo prevenirlas. Madrid: CEAPA.

Roca, M. (2002). Autoeficacia: su valor para la psicoterapia cognitivo conductual,. Revista cubana de psicología, vol. 19, núm. 3, 195-200. 
UNODC. (2017, Junio). III Estudio epidemiológico andino sobre consumo de drogas en la población universitaria de Ecuador. Obtenido de Oficina de las Naciones Unidas contra la Droga y el Delito: https://www.unodc.org/documents/peruandecuador/Publicaciones/Publicacion es2017/Informe_Universitarios_Ecuador.pdf

Villamarín, F. (1990). Papel de la auto-eficacia en los trastornos de ansiedad y depresión. Análisis y Modificación de Conducta, Vol. 16, núm. 47, 55-79.

Witkiewitz, K., \& Marlatt, G. (2004). Prevención de recaídas para problemas de alcohol y drogas: eso era Zen, esto es Tao. Am Psychol, vol. 59, núm. 4, 224235. 